\title{
Scaling Lattice QCD beyond 100 GPUs
}

\author{
R. Babich ${ }^{*}$ \\ Center for Computational \\ Science \\ Boston University \\ Boston, MA 02215, USA \\ rbabich@bu.edu \\ G. Shi \\ National Center for \\ Supercomputing Applications \\ University of Illinois \\ Urbana, IL 61801 \\ gshi@ncsa.uiuc.edu
}

\author{
M. A. Clark \\ Harvard-Smithsonian Center \\ for Astrophysics \\ 60 Garden St \\ Cambridge, MA 02143, USA \\ mikec@seas.harvard.edu
}

\author{
R. C. Brower \\ Center for Computational \\ Science \\ Boston University \\ Boston, MA 02215, USA \\ brower@bu.edu
}

\author{
B. Joó ${ }^{*}$ \\ Thomas Jefferson National \\ Accelerator Facility \\ Newport News, VA 23606, \\ USA \\ bjoo@jlab.org
}

\author{
S. Gottlieb \\ Department of Physics \\ Indiana University \\ Bloomington, IN 47405, USA \\ sg@indiana.edu
}

\begin{abstract}
Over the past five years, graphics processing units (GPUs) have had a transformational effect on numerical lattice quantum chromodynamics (LQCD) calculations in nuclear and particle physics. While GPUs have been applied with great success to the post-Monte Carlo "analysis" phase which accounts for a substantial fraction of the workload in a typical LQCD calculation, the initial Monte Carlo "gauge field generation" phase requires capability-level supercomputing, corresponding to $\mathrm{O}(100)$ GPUs or more. Such strong scaling has not been previously achieved. In this contribution, we demonstrate that using a multi-dimensional parallelization strategy and a domain-decomposed preconditioner allows us to scale into this regime. We present results for two popular discretizations of the Dirac operator, Wilson-clover and improved staggered, employing up to 256 GPUs on the Edge cluster at Lawrence Livermore National Laboratory.
\end{abstract}

\section{Categories and Subject Descriptors}

G.1.3 [Numerical Linear Algebra]: Linear systems (direct and iterative methods; G.1.8 [Partial Differential Equations]: Domain decomposition methods, Finite difference methods, Iterative solution techniques; J.2 [Physical Sciences and Engineering]: Physics

\section{Keywords}

Lattice QCD, GPU, Krylov solvers, domain decomposition

*These authors contributed equally to this work.

Permission to make digital or hard copies of all or part of this work for personal or classroom use is granted without fee provided that copies are not made or distributed for profit or commercial advantage and that copies bear this notice and the full citation on the first page. To copy otherwise, to republish, to post on servers or to redistribute to lists, requires prior specific permission and/or a fee.

SC11 November 12-18, 2011, Seattle, Washington, USA

Copyright 2011 ACM 978-1-4503-0771-0/11/11 ...\$10.00.

\section{INTRODUCTION}

Lattice QCD (LQCD) is one of the original computational grand challenges 1]. Increasingly accurate numerical solutions of this quantum field theory are being used in tandem with experiment and observation to gain a deeper quantitative understanding for a range of phenomena in nuclear and high energy physics. Advances during the last quarter century required prodigious computational power, the development of sophisticated algorithms, and highly optimized software. As a consequence LQCD is one of the driver applications that have stimulated the evolution of new architectures such as the BlueGene series 22. Graphics processing unit (GPU) clusters challenge us to adapt lattice field theory software and algorithms to exploit this potentially transformative technology. Here we present methods allowing QCD linear solvers to scale to hundreds of GPUs with high efficiency. The resulting multi-teraflop performance is now comparable to typical QCD codes running on capability machines such as the Cray and the BlueGene/P using several thousand cores.

GPU computing has enjoyed a rapid growth in popularity in recent years due to the impressive performance to price ratio offered by the hardware and the availability of free software development tools and example codes. Currently, the fastest supercomputer in the world, Tianhe-1A, is a GPU-based system, and several large-scale GPU systems are either under consideration or are in active development. Examples include the Titan system proposed for the Oak Ridge Leadership Computing Facility (OLCF) and the NSF Track 2 Keeneland system to be housed at the National Institute for Computational Sciences (NICS).

Such systems represent a larger trend toward heterogeneous architectures, characterized not only by multiple processor types (GPU and conventional CPU) with very different capabilities, but also by a deep memory hierarchy exhibiting a large range of relevant bandwidths and latencies. These features are expected to typify at least one path (or "swim-lane") toward exascale computing. The intrinsic imbalance between different subsystems can present bottlenecks and a real challenge to application developers. In particular, the PCI-E interface currently used to connect 
CPU, GPU, and communications fabric on commodity clusters can prove a severe impediment for strong-scaling the performance of closely coupled, nearest-neighbor stencil-like codes into the capability computing regime. Overcoming such limitations is vital for applications to succeed on future large-scale heterogeneous resources.

We consider the challenge of scaling LQCD codes to a large number of GPUs. LQCD is important in high energy and nuclear physics as it is the only currently known firstprinciples non-perturbative approach for calculations involving the strong force. Not only is LQCD important from the point of view of physics research, but historically LQCD codes have often been used to benchmark and test large scale computers. The balanced nature of QCD calculations, which require approximately 1 byte/flop in single precision, as well as their regular memory access and nearest neighbor communication patterns have meant that LQCD codes could be deployed quickly, scaled to large partitions, and used to exercise the CPU, memory system, and communications fabric. In GPU computing, LQCD has been highly successful in using various forms of data compression and mixed precision solvers 3 to alleviate memory bandwidth contraints on the GPU in order to attain high performance. Our multiGPU codes 4, 5, 6] are in production, performing capacity analysis calculations on systems at several facilities, including Lincoln and EcoG (NCSA), Longhorn (TACC), the "9g" and "10g" clusters at Jefferson Laboratory (JLab), and Edge (LLNL).

The challenge is now to scale LQCD computations into the $\mathrm{O}(100)$ GPU regime, which is required if large GPU systems are to replace the more traditional massively parallel multicore supercomputers that are used for the gauge generation step of LQCD. Here, capability-class computing is required since the algorithms employed consist of single streams of Monte Carlo Markov chains, and so require strong scaling.

Our previous multi-GPU implementations utilized a strategy of parallelizing in the time direction only, using traditional iterative Krylov solvers (e.g., conjugate gradients). This severely limits the number of GPUs that can be used and thus maximum performance. In order to make headway, it has become important to parallelize in additional dimensions in order to give sublattices with improved surfaceto-volume ratios and to explore algorithms that reduce the amount of communication altogether such as domain decomposed approaches.

In this paper, we make the following contributions: (i) we parallelize the application of the discretized Dirac operator in the QUDA library to communicate in multiple dimensions, (ii) we investigate the utility of an additive Schwarz domain-decomposed preconditioner for the Generalized Conjugate Residual (GCR) solver, and (iii) we perform performance tests using (i) and (ii) on partitions of up to 256 GPUs. The paper is organized as follows: we present an outline of LQCD computations in Sec. 2 and discuss iterative linear solvers in Sec. 3. A brief overview of previous and related work is given in Sec. 4, and in Sec. 5 we describe the QUDA library upon which this work is based. The implementation of the multi-dimensional, multi-GPU parallelization is discussed in Sec. 6] The construction of our optimized linear solvers are elaborated in Sec. 8 . We present performance results in Sec. 9 Finally, we summarize and conclude in Sec. 10 .

\section{LATTICE QCD}

Weakly coupled field theories such as quantum electrodynamics can by handled with perturbation theory. In QCD, however, at low energies perturbative expansions fail and a non-perturbative method is required. Lattice QCD is the only known, model independent, non-perturbative tool currently available to perform QCD calculations.

LQCD calculations are typically Monte-Carlo evaluations of a Euclidean-time path integral. A sequence of configurations of the gauge fields is generated in a process known as configuration generation. The gauge configurations are importance-sampled with respect to the lattice action and represent a snapshot of the QCD vacuum. Configuration generation is inherently sequential as one configuration is generated from the previous one using a stochastic evolution process. Many variables can be updated in parallel and the focused power of capability computing systems has been essential. Once the field configurations have been generated, one moves on to the second stage of the calculation, known as analysis. In this phase, observables of interest are evaluated on the gauge configurations in the ensemble, and the results are then averaged appropriately, to form ensemble averaged quantities. It is from the latter that physical results such as particle energy spectra can be extracted. The analysis phase can be task parallelized over the available configurations in an ensemble and is thus extremely suitable for capacity level work on clusters, or smaller partitions of supercomputers.

\subsection{Dirac PDE discretization}

The fundamental interactions of QCD, those taking place between quarks and gluons, are encoded in the quark-gluon interaction differential operator known as the Dirac operator. A proper discretization of the Dirac operator for lattice QCD requires special care. As is common in PDE solvers, the derivatives are replaced by finite differences. Thus on the lattice, the Dirac operator becomes a large sparse matrix, $M$, and the calculation of quark physics is essentially reduced to many solutions to systems of linear equations given by

$$
M x=b .
$$

Computationally, the brunt of the numerical work in LQCD for both the gauge generation and analysis phases involves solving such linear sytems.

A small handful of discretizations are in common use, differing in their theoretical properties. Here we focus on two of the most widely-used forms for $M$, the SheikholeslamiWohlert 7] form (colloquially known as Wilson-clover), and the improved staggered form, specifically the $a^{2}$ tadpoleimproved (asqtad) formulation 8.

\subsection{Wilson-clover matrix}

The Wilson-clover matrix is a central-difference discretization of the Dirac operator, with the addition of a diagonallyscaled Laplacian to remove the infamous fermion doublers (which arise due to the red-black instability of the centraldifference approximation). When acting in a vector space that is the tensor product of a 4-dimensional discretized Eu- 
clidean spacetime, spin space, and color space it is given by

$$
\begin{aligned}
M_{x, x^{\prime}}^{W C}= & -\frac{1}{2} \sum_{\mu=1}^{4}\left(P^{-\mu} \otimes U_{x}^{\mu} \delta_{x+\hat{\mu}, x^{\prime}}+P^{+\mu} \otimes U_{x-\hat{\mu}}^{\mu \dagger} \delta_{x-\hat{\mu}, x^{\prime}}\right) \\
& +\left(4+m+A_{x}\right) \delta_{x, x^{\prime}} \\
\equiv & -\frac{1}{2} D_{x, x^{\prime}}^{W C}+\left(4+m+A_{x}\right) \delta_{x, x^{\prime}}
\end{aligned}
$$

Here $\delta_{x, y}$ is the Kronecker delta; $P^{ \pm \mu}$ are $4 \times 4$ matrix projectors in spin space; $U$ is the QCD gauge field which is a field of special unitary $3 \times 3$ (i.e., $\mathrm{SU}(3))$ matrices acting in color space that live between the spacetime sites (and hence are referred to as link matrices); $A_{x}$ is the $12 \times 12$ clover matrix field acting in both spin and color space ${ }^{1}$ corresponding to a first order discretization correction; and $m$ is the quark mass parameter. The indices $x$ and $x^{\prime}$ are spacetime indices (the spin and color indices have been suppressed for brevity). This matrix acts on a vector consisting of a complex-valued 12-component color-spinor (or just spinor) for each point in spacetime. We refer to the complete lattice vector as a spinor field.

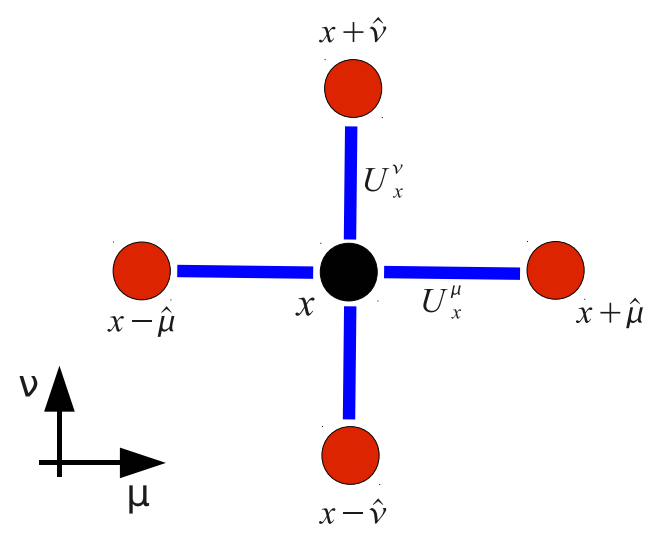

Figure 1: The nearest neighbor stencil part of the lattice Dirac operator $D$, as defined in (2), in the $\mu-\nu$ plane. The color-spinor fields are located on the sites. The $\mathrm{SU}(3)$ color matrices $U_{x}^{\mu}$ are associated with the links. The nearest neighbor nature of the stencil suggests a natural even-odd (red-black) coloring for the sites.

\subsection{Improved staggered matrix}

The staggered matrix again is a central-difference discretization of the Dirac operator; however, the fermion doublers are removed through "staggering" the spin degrees of freedom onto neighboring lattice sites. This essentially reduces the number of spin degrees of freedom per site from four to one, which reduces the computational burden significantly. This transformation, however, comes at the expense of increased discretization errors, and breaks the so-called quark-flavor symmtery. To reduce these discretization errors, the gauge field that connects nearest neighboring sites on the lattice ( $U_{x}^{\mu}$ in Equation 2 ) is smeared, which essen-

\footnotetext{
${ }^{1}$ Each clover matrix has a Hermitian block diagonal, antiHermitian block off-diagonal structure, and can be fully described by 72 real numbers.
}

tially is a local averaging of the field. There are many prescriptions for this averaging; and here we employ the popular asqtad procedure 8]. The errors are further reduced through the inclusion of the third neighboring spinors in the derivative approximaton. The asqtad matrix is given by

$$
\begin{aligned}
& M_{x, x^{\prime}}^{I S}=-\frac{1}{2} \sum_{\mu=1}^{4}\left(\hat{U}_{x}^{\mu} \delta_{x+\hat{\mu}, x^{\prime}}+\hat{U}_{x-\hat{\mu}}^{\mu \dagger} \delta_{x-\hat{\mu}, x^{\prime}}+\right. \\
&\left.\check{U}_{x}^{\mu} \delta_{x+3 \hat{\mu}, x^{\prime}}+\check{U}_{x-3 \hat{\mu}}^{\mu \dagger} \delta_{x-3 \hat{\mu}, x^{\prime}}\right)+m \delta_{x, x^{\prime}} \\
& \equiv-\frac{1}{2} D_{x, x^{\prime}}^{I S}+m \delta_{x, x^{\prime}} .
\end{aligned}
$$

Unlike $M^{W C}$, the matrix $M^{I S}$ consists solely of a derivative term $D^{I S}$ and the mass term. There are two gauge fields present: $\hat{U}_{x}^{\mu}$ is the fat gauge field, and is the field produced from locally averaging $U_{x}^{\mu}$; and $\check{U}_{x}^{\mu}$ is the long gauge field produced by taking the product of the links $U_{x}^{\mu} U_{x+\hat{\mu}}^{\mu} U_{x+2 \hat{\mu}}^{\mu}$. While both of these fields are functions of the original field $U_{x}^{\mu}$, in practice, these fields are precalculated before the application of $M_{x, x^{\prime}}^{I S}$ since iterative solvers will require the application of $M_{x, x^{\prime}}^{I S}$ many hundreds or thousands of times. Since there are no separate spin degrees of freedom at each site, this matrix acts on a vector of complex-valued 3-component colors; however, for convenience we nevertheless refer to the complex lattice vector as a spinor field.

\section{ITERATIVE SOLVERS}

\subsection{Krylov solvers}

For both discretizations under consideration, $M$ is a large sparse matrix, and iterative Krylov solvers are typically used to obtain solutions to Equation (1), requiring many repeated evaluations of the sparse matrix-vector product. The Wilsonclover matrix is non-Hermitian, so either Conjugate Gradients 9] on the normal equations (CGNE or CGNR) is used, or more commonly, the system is solved directly using a non-symmetric method, e.g., BiCGstab 10]. Even-odd (also known as red-black) preconditioning is almost always used to accelerate the solution finding process for this system, where the nearest neighbor property of the $D^{W C}$ matrix is exploited to solve the Schur complement system [1].

The staggered fermion matrix is anti-Hermitian, and has the convenient property that when multiplied by its Hermitian conjugate, the even and odd lattices are decoupled and can be solved independently from each other. There are no commonly used preconditioners for the staggered matrix. When simulating asqtad fermions, for both the gauge field generation and for the analysis stages, one is confronted with solving problems of the form

$$
\left(M^{\dagger} M+\sigma_{i} I\right) x_{i}=b \quad i=1 \ldots N
$$

where $\sigma_{i}$ is a constant scalar and $I$ is the identity matrix. This is equivalent to solving $N$ different linear systems at different mass parameters for a constant source $b$. Since the generated Krylov spaces are the same for each of these linear systems, one can use a multi-shift solver (also known as a multi-mass solver) to produce all $N$ solutions simultaneously in the same number of iterations as the smallest shift (least well conditioned) 12].

In both cases, the quark mass controls the condition number of the matrix, and hence the convergence of such iter- 
ative solvers. Unfortunately, physical quark masses correspond to nearly indefinite matrices. Given that current lattice volumes are at least $10^{8}$ degrees of freedom in total, this represents an extremely computationally demanding task. For both the gauge generation and analysis stages, the linear solver accounts for $80-99 \%$ of the execution time.

\subsection{Additive Schwarz preconditioner}

As one scales lattice calculations to large core counts, on leadership-class partitions, one is faced with a strongscaling challenge: as core counts increase, for a fixed global lattice volume the local sub-volume per core decreases and the surface-to-volume ratio of the local sub-lattice increases. Hence, the ratio of communication to local computation also grows. For sufficiently many cores, it becomes impossible to hide communication by local computation and the problem becomes communications bound, at the mercy of the system interconnect. This fact, with the need for periodic global reduction operations in the Krylov solvers, leads to a slowdown of their performance in the large scaling limit. For GPU-based clusters, where inter-GPU communication is gated by the PCI-E bus, this limitation is substantially more pronounced and can occur in partitions as small as $\mathrm{O}(10)$ GPUs or less.

This challenge of strong scaling of traditional Krylov solvers motivates the use of solvers which minimize the amount of communication. Such solvers are commonly known as domain-decomposition solvers and two forms of them are commonly used: multiplicative Schwarz and additive Schwarz processes 13. In this work we focus upon the additive Schwarz method. Here, the entire domain is partitioned into blocks which may or may not overlap. The system matrix is then solved within these blocks, imposing Dirichlet (zero) boundary conditions at the block boundaries. The imposition of Dirichlet boundary conditions means that no communication is required between the blocks, and that each block can be solved independently. It is therefore typical to assign the blocks to match the sub-domain assigned to each processor in a parallel decomposition of a domain. A tunable parameter in these solvers is the degree of overlap of the blocks, with a greater degree of overlap corresponding to increasing the size of the blocks, and hence the amount of computation required to solve each block. A larger overlap will typically lead to requiring fewer iterations to reach convergence, since, heuristically, the larger sub blocks, will approximate better the original matrix and hence their inverses will form better preconditioners. Note that an additive Schwarz solver with non-overlapping blocks is equivalent to a block-Jacobi solver.

Typically, Schwarz solvers are not used as a standalone solver, but rather they are employed as preconditioners for an outer Krylov method. Since each local system matrix is usually solved using an iterative solver, this requires that the outer solver be a flexible solver. Generalized conjugate residual (GCR) is such a solver, and we shall employ it for the work in this paper.

\section{OVERVIEW OF RELATED WORK}

Lattice QCD calculations on GPUs were originally reported in 14] where the immaturity of using GPUs for general purpose computation necessitated the use of graphics APIs. Since the advent of CUDA in 2007, there has been rapid uptake by the LQCD community (see 15] for an overview). More recent work includes [16, which targets the computation of multiple systems of equations with Wilson fermions where the systems of equations are related by a linear shift. Solving such systems is of great utility in implementing the overlap formulation of QCD. This is a problem we target in the staggered-fermion solver below. The work in 17 targets the domain-wall fermion formulation of LQCD. This work concerns the QUDA library 3, which we describe in Sec. 5 below.

Most work to date has concerned single-GPU LQCD implementations, and beyond the multi-GPU parallelization of QUDA [4, 6] and the work in [18, which targets a multi-GPU implementation of the overlap formulation, there has been little reported in the literature, though we are aware of other implementations which are in production [19.

Domain-decomposition algorithms were first introduced to LQCD in 20], through an implementation of the Schwarz Alternating Procedure preconditioner, which is a multiplicative Schwarz preconditioner. More akin to the work presented here is the work in 21] where a restricted additive Schwarz preconditioner was implemented for a GPU cluster. However, the work reported in 21] was carried out on a rather small cluster containing only 4 nodes and connected with Gigabit Ethernet. The work presented here aims for scaling to $\mathrm{O}(100)$ GPUs using a QDR Infiniband interconnect.

\section{QUDA}

The QUDA library is a package of optimized CUDA kernels and wrapper code for the most time-consuming components of an LQCD computation. It has been designed to be easy to interface to existing code bases, and in this work we exploit this interface to use the popular LQCD applications Chroma and MILC. The QUDA library has attracted a diverse developer community of late and is being used in production at LLNL, Jlab and other U.S. national laboratories, as well as in Europe. The latest development version is always available in a publically-accessible source code repository 22.

QUDA implements optimized linear solvers, which when running on a single GPU achieve up to $24 \%$ of the GPU's peak performance through aggressive optimization. The general strategy is to assign a single GPU thread to each lattice site, each thread is then responsible for all memory traffic and operations required to update that site on the lattice given the stencil operator. Maximum memory bandwidth is obtained by reordering the spinor and gauge fields to achieve memory coalescing using structures of float2 or float4 arrays, and using the texture cache where appropriate. Memory traffic reduction is employed where possible to overcome the relatively low arithmetic intensity of the Dirac matrix-vector operations, which would otherwise limit performance. Strategies include: (a) using compression for the $S U(3)$ gauge matrices to reduce the 18 real numbers to 12 (or 8) real numbers at the expense of extra computation; (b) using similarity transforms to increase the sparsity of the Dirac matrices; (c) utilizing a custom 16-bit fixed-point storage format (here on referred to as half precision) together with mixed-precision linear solvers to achieve high speed with no loss in accuracy.

Other important computational kernels provided in the library include gauge field smearing routines for constructing the fat gauge field used in the asqtad variant of the improved 
staggered discretization, as well as force term computations required for gauge field generation.

The extension of QUDA to support multiple GPUs was reported in [4], where both strong and weak scaling was performed on up to 32 GPUs using a lattice volume of $32^{3} \times 256$ with Wilson-clover fermions. This employed partitioning of the lattice along the time dimension only, and was motivated by expediency and the highly asymmetric nature of the lattices being studied. While this strategy was sufficient to achieve excellent (artificial) weak scaling performance, it severely limits the strong scaling achievable for realistic volumes because of the increase in surface-to-volume ratio. The application of these strategies to the improved staggered discretization was described in 6], where strong scaling was achieved on up to 8 GPUs using a lattice volume of $24^{3} \times 96$. Here the single dimensional parallelization employed restricts scaling more severely than for Wilson-clover because of the 3-hop stencil of the improved staggered operator which decreases the locality of the operator.

\section{MULTI-DIMENSIONAL PARTITIONING}

\subsection{General strategy}

Because lattice discretizations of the Dirac operator generally only couple sites of the lattice that are nearby in spacetime, the first step in any parallelization strategy is to partition the lattice. As indicated above, prior to this work multi-GPU parallelization of the QUDA library had been carried out with the lattice partitioned along only a single dimension. The time $(T)$ dimension was chosen, first because typical lattice volumes are asymmetric with $T$ longest, and secondly because this dimension corresponds to the slowest varying index in memory in our implementation, making it possible to transfer the boundary face from GPU to host with a straightforward series of memory copies. Going beyond this approach requires much more general handling of data movement, computation, and synchronization, as we explore here.

In the general case, upon partitioning the lattice each GPU is assigned a 4-dimensional subvolume that is bounded by at most eight 3-dimensional "faces." Updating sites of the spinor field on or near the boundary requires data from neighboring GPUs. The data received from a given neighbor is stored in a dedicated buffer on the GPU which we will refer to as a "ghost zone" (since it shadows data residing on the neighbor). Computational kernels are modified so as to be aware of the partitioning and read data from the appropriate location - either from the array corresponding to the local subvolume or one of the ghost zones. Significant attention is paid to maintaining memory coalescing and avoiding thread divergence, as detailed below.

Ghost zones for the spinor field are placed in memory after the local spinor field so that BLAS-like routines, including global reductions, may be carried out efficently. While the ghost spinor data for the $\mathrm{T}$ dimension is contiguous and can be copied without a gather operation, the ghost spinor data for the other three dimensions must be collected into contiguous GPU memory buffers by a GPU kernel before it can be transfered to host memory. The ghost zone buffers are then exchanged between neighboring GPUs (possibly residing in different nodes). Once inter-GPU communication is complete, the ghost zones are copied to locations adjoining the local array in GPU memory. Allocation of ghost zones

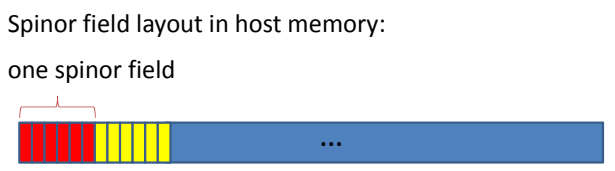

Spinor field layout in GPU memory:

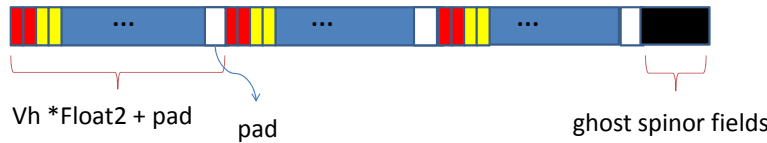

Figure 2: Spinor field layout in host and GPU memory for the staggered discretization (consisting of 6 floating point numbers per site). Here $\mathrm{Vh}$ is half the local volume of the lattice, corresponding to the number of sites in an even/odd subset. Layout for the Wilson-clover discretization is similar, wherein the spinor field consists of 24 floating point numbers per site.

and data exchange in a given dimension only takes place when that dimension is partitioned, so as to ensure that GPU memory as well as PCI-E and interconnect bandwidth are not wasted. Layout of the local spinor field, ghost zones, and padding regions are shown in Fig. 2 The padding region is of adjustable length and serves to reduce partition camping 3, 23 on previous-generation NVIDIA GPUs ${ }^{2}$ The gauge field is allocated with a similar padding region, and we use this space to store ghost zones for the gauge field, which must only be transfered once at the beginning of a solve. The layout is illustrated in Fig. 3

For communication, our implementation is capable of employing either of two message-passing frameworks - MPI or QMP. The latter "QCD message-passing" standard was originally developed to provide a simplified subset of communication primitives most used by LQCD codes, allowing for optimized implementations on a variety of architectures, including purpose-built machines that lack MPI. Here we rely on the reference implementation, which serves as a thin layer over MPI itself (but nevertheless serves a purpose as the communications interface used natively by Chroma, for example). Accordingly, performance with the two frameworks is virtually identical. At present, we assign GPUs to separate processes which communicate via message-passing. Exploration of peer-to-peer memory copies, recently added in CUDA 4.0, and host-side multi-threading is underway.

\subsection{Interior and exterior kernels}

In [4, 6], where only the time dimension of the lattice was

\footnotetext{
${ }^{2}$ This is less a concern for the Tesla M2050 cards used in this study, as the Fermi memory controller employs address hashing to alleviate this problem.
} 
Gauge field layout in host memory:

one gauge field

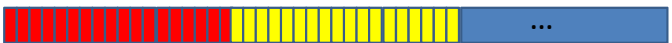

Gauge field layout in GPU memory:

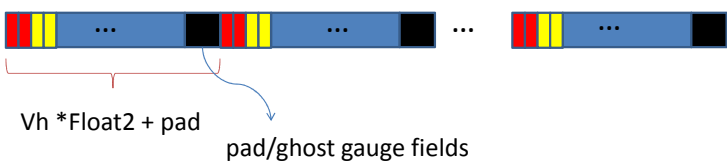

Figure 3: Gauge field layout in host and GPU memory. The gauge field consists of 18 floating point numbers per site (when no reconstruction is employed) and is ordered on the GPU so as to ensure that memory accesses in both interior and boundary-update kernels are coalesced to the extent possible.

partitioned, we separated the application of the Dirac operator into two kernels, one to update sites on the boundaries of the local sublattice (the exterior kernel) and one to perform all remaining work (the interior kernel). Here we extend this approach by introducing one exterior kernel for every dimension partitioned, giving a total of four exterior kernels in the most general case. The interior kernel executes first and computes the spinors interior to the subvolume, as well as any contributions to spinors on the boundaries that do not require data from the ghost zones. For example, if a spinor is located only on the $\mathrm{T}+$ boundary, the interior kernel computes contributions to this spinor from all spatial dimensions, as well as that of the negative $\mathrm{T}$ direction. The contribution from the positive $\mathrm{T}$ direction will be computed in the $\mathrm{T}$ exterior kernel using the ghost spinor and gauge field from the $\mathrm{T}+$ neighbor. Since spinors on the corners belong to multiple boundaries, they receive contributions from multiple exterior kernels. This introduces a data dependency between exterior kernels, which must therefore be executed sequentially.

Another consideration is the ordering used for assigning threads to sites. For the interior kernel and $\mathrm{T}$ exterior kernel, the one-dimensional thread index (given in CUDA $\mathrm{C}$ by (blockIdx. $\mathrm{x} *$ blockDim.x + threadIdx.x)) is assigned to sites of the four-dimensional sublattice in the same way that the spinor and gauge field data is ordered in memory, with $\mathrm{X}$ being the fastest varying index and $\mathrm{T}$ the slowest. It is thus guaranteed that all spinor and gauge field accesses are coalesced. In the X,Y,Z exterior kernels, however, only the destination spinors are indexed in this way, while the ghost spinor and gauge field are indexed according to a different mapping. This makes it impossible to guarantee coalescing for both reads and writes; one must choose one order or the other for assigning the thread index. We choose to employ the standard T-slowest mapping for the X,Y,Z exterior kernels to minimize the penalty of uncoalesced accesses, since a greater fraction of the data traffic comes from the gauge field and source spinors.

\subsection{Computation, communication, and streams}

Our implementation employs CUDA streams to overlap computation with communication, as well as to overlap GPUto-host with inter-node communication. Two streams per dimension are used, one for gathering and exchanging spinors in the forward direction and the other in the backward direction. One additional stream is used for executing the interior and exterior kernels, giving a total of 9 streams as shown in Fig. 4. The gather kernels for all dimensions are launched on the GPU immediately so that communication in all directions can begin. The interior kernel is executed after all gather kernels finish, overlapping completely with the communication. We use different streams for different dimensions so that the different communication components can overlap with each other, including the device-to-host memory copy, the copy from pinned host memory to pagable host memory, the MPI send and receive, the memory copy from pagable memory to pinned memory on the receiving side, and the final host-to-device memory copy. While the interior kernel can be overlapped with communications, the exterior kernels must wait for arrival of the ghost data. As a result, the interior kernel and subsequent exterior kernels are placed in the same stream, and each exterior kernel blocks waiting for communication in the corresponding dimension to finish. For small subvolumes, the total communication time over all dimensions is likely to exceed the interior kernel run time, resulting in some interval when the GPU is idle (see Fig. 4) and thus degrading overall performance.

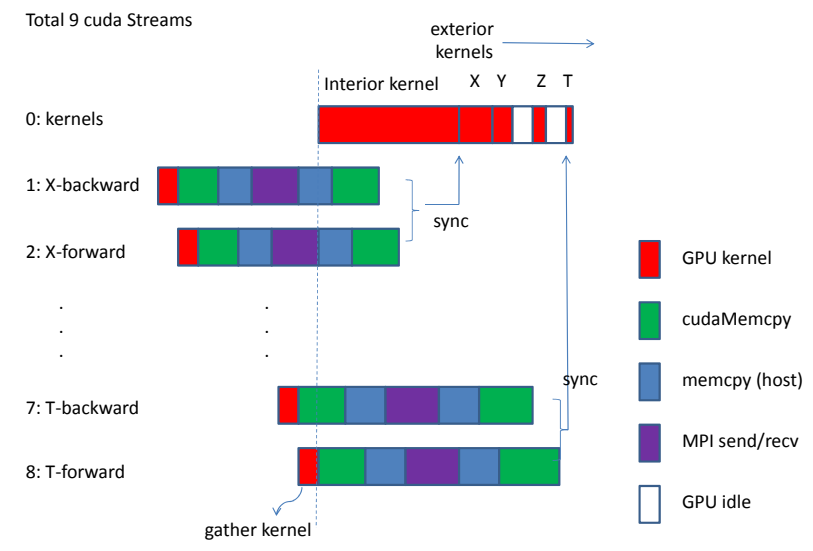

Figure 4: Usage of CUDA streams in the application of the Dirac operator, illustrating the multiple stages of communication. A single stream is used for the interior and exterior kernels, and two streams per dimension are used for gather kernels, PCI-E data transfer, host memory copies, and inter-node communication.

When communicating over multiple dimensions with small 
subvolumes, the communication cost dominates over computation, and so any reduction in the communication is likely to improve performance. The two host memory copies are required due to the fact that GPU pinned memory is not compatible with memory pinned by MPI implementations; GPU-Direct 24 was not readily available on the cluster used in this study. We expect to be able to remove these extra memory copies in the future when better support from GPU and MPI vendors is forthcoming. CUDA 4.0, recently released, includes a promising GPU-to-GPU direct communication feature that we will explore in the future to further reduce the communication cost.

\section{DIRAC OPERATOR PERFORMANCE}

\subsection{Hardware description}

For the numerical experiments discussed in this paper we used the Edge visualization cluster installed at Lawrence Livermore National Laboratory. Edge is comprised of a total of 216 nodes, of which 206 are compute nodes available for batch jobs. Each compute node is comprised of dual-socket six-core Intel X5660 Westmere CPUs running at $2.8 \mathrm{GHz}$ and two NVIDIA Tesla M2050 GPUs, running with error correction (ECC) enabled. The two GPUs share a single x16 PCI-E connection to the I/O hub (IOH) via a switch. Eight of the remaining PCI-E lanes serve a quad data rate (QDR) InfiniBand interface which can thus run at full bandwidth. The compute nodes run a locally maintained derivative of a CentOS 5 kernel with revision 2.6.18-chaos103.

To build and run our software we used OpenMPI version 1.5 built on top of the system $\mathrm{GNU} \mathrm{C} / \mathrm{C}++$ compiler version 4.1.2. To build and link against the QUDA library we used release candidate 1 of CUDA version 4.0 with driver version 270.27 .

\subsection{Wilson-clover}

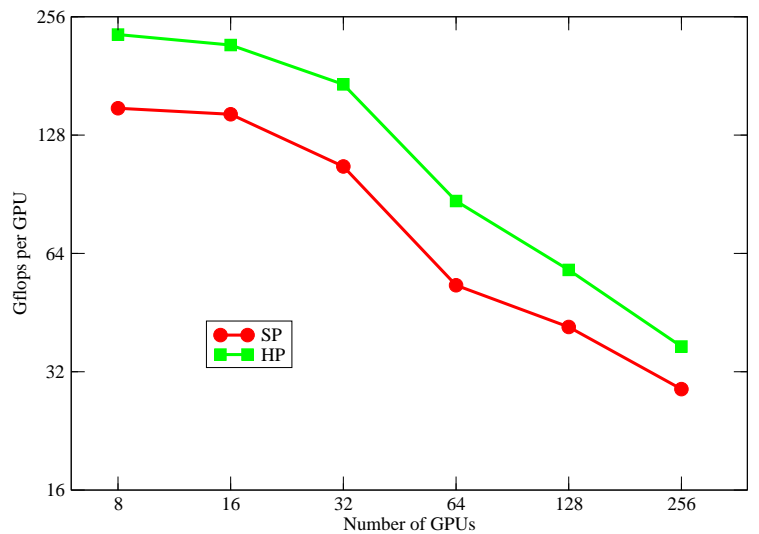

Figure 5: Strong-scaling results for the Wilsonclover operator in single (SP) and half (HP) precisions ( $V=32^{3} \times 256,12$ gauge reconstruction).

In Fig. 5 we show the strong scaling of the Wilson-clover operator on up to 256 GPUs. We see significant departures from ideal scaling for more than 32 GPUs, as increasing the surface-to-volume ratio increases the amount of time spent in communication, versus computation. It seems that, for more than 32 GPUs, we are no longer able to sufficiently overlap computation with communication, and the implementation becomes communications bound. We note also that as the communications overhead grows, the performance advantage of the half precision operator over the single precision operator appears diminished. The severity of the scaling violations seen here highlights the imbalance between the communications and compute capability of GPU clusters. To overcome this constraint, algorithms which reduce communication, such as the domain-decomposition algorithms described below, are absolutely essential.

\subsection{Improved staggered}

In Fig. 6. we plot the performance per GPU for the asqtad volume used in this study. A number of interesting observations can be made about this plot. At a relatively low number of GPUs, where we are less communications-bound, having faster kernel performance is more important than the optimal surface-to-volume ratio. As the number of GPUs is increased, the minimization of the surface-to-volume ratio becomes increasingly important, and the XYZT partitioning scheme, which has the worst single-GPU performance, obtains the best performance on 256 GPUs.

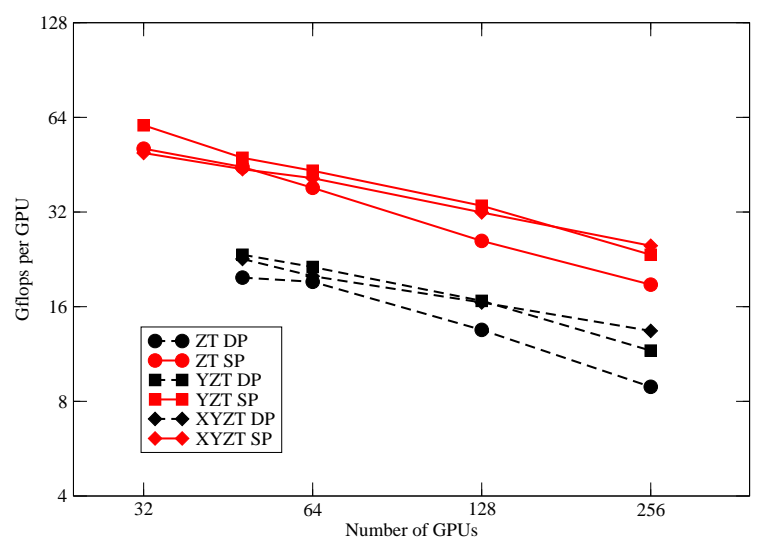

Figure 6: Strong-scaling performance for the asqtad operator in double (DP) and single (SP) precision. The legend labels denote which dimensions are partitioned between GPUs $\left(V=64^{3} \times 192\right.$, no gauge reconstruction).

\section{BUILDING SCALABLE SOLVERS}

\subsection{Wilson-clover additive Schwarz precondi- tioner}

The poor scaling of the application of the Wilson-clover matrix at a high number of GPUs at this volume motivates 
the use of communication-reducing algorithms. In this initial work, we investigate using a non-overlapping additive Schwarz preconditioner for GCR. In the text that follows, we refer to this algorithm as GCR-DD.

Implementation of the preconditioner is simple: essentially, we just have to switch off the communications between GPUs. This means that in applying the Dirac operator, the sites that lie along the communication boundaries only receive contributions from sites local to that node. Additionally, since the solution in each domain is independent from every other, the reductions required in each of the domainspecific linear solvers are restricted to that domain only. As a result the solution of the preconditioner linear system will operate at similar efficiency to the equivalent single-GPU performance at this local volume. The imposition of the Dirichlet boundary conditions upon the local lattice leads to a vastly reduced condition number. This, coupled with the fact that only a very loose approximation of the local system matrix is required, means that only a small number of steps of minimum residual (MR) are required to achieve satisfactory accuracy.

The outer GCR solver builds a Krylov subspace, within which the residual is minimized and the corresponding solution estimate is obtained. Unlike solvers with explicit recurrence relations, e.g., CG, the GCR algorithm for the general matrix problem requires explicit orthogonalization at each step with respect to the previously generated Krylov space. Thus, the size of the Krylov space is limited by the computational and memory costs of orthogonalization. After the limit of the Krylov space has been reached, the algorithm is restarted, and the Krylov space is rebuilt.

Similar to what was reported for BiCGstab 3], we have found that using mixed precision provides significant acceleration of the GCR-DD solver algorithm. We exclusively use half precision for solving the preconditioned system. This is natural since only a very loose approximation is required. Additionally, the restarting mechanism of GCR provides a natural opportunity for using mixed precision: the Krylov space is built up in low precision and restarted in high precision. This approach also conserves the limited GPU memory, allowing for larger Krylov spaces to be built, albeit in lower precision. We follow the implicit solution update scheme described in 20] since this reduces the orthogonalization overhead. For the physics of interest, the inherent noise present in the Monte Carlo gauge generation process is such that single-precision accuracy is sufficient. Thus we have found best performance using a single-half-half solver, where the GCR restarting is performed in single precision, the Krylov space construction and accompanying orthogonalization is done in half precision, and the preconditioner is solved in half precision. In minimizing the residual in half precision, there is the inherent risk of the iterated residual straying too far from the true residual. Thus, we have added an early termination criteria for the Krylov subspace generation, where if the residual is decreased by more than a given tolerance $\delta$ from the start of the Krylov subspace generation, the algorithm is restarted. The mixed-precision GCR-DD solver is illustrated in Algorithm 1

\subsection{Improved staggered}

While using a multi-shift CG solver can lead to significant speedups on traditional architectures, their use is less clearcut on GPUs: multi-shift solvers cannot be restarted, mean-

$$
\begin{aligned}
& k=0 \\
& r_{0}=b-M x_{0} \\
& \hat{r}_{0}=r_{0} \\
& \hat{x}=0 \\
& \text { while }\left\|r_{0}\right\|>\text { tol } \text { do } \\
& \hat{p}_{k}=\hat{K} \hat{r}_{k} \\
& \hat{z}_{k}=\hat{M} \hat{p}_{k} \\
& \text { // Orthogonalization } \\
& \text { for } i \leftarrow 0 \text { to } k-1 \text { do } \\
& \beta_{i, k}=\left(\hat{z}_{i}, \hat{z}_{k}\right) \\
& \hat{z}_{k}=\hat{z}_{k}-\beta_{i, k} \hat{z}_{i} \\
& \text { end } \\
& \gamma_{k}=\left\|\hat{z}_{k}\right\| \\
& z_{k}=z_{k} / \gamma_{k} \\
& \alpha_{k}=\left(\hat{z}_{k}, \hat{r}_{k}\right) \\
& \hat{r}_{k+1}=\hat{r}_{k}-\alpha_{k} \hat{z}_{k} \\
& k=k+1 \\
& \text { // High precision restart } \\
& \text { if } k=k_{\max } \text { or }\left\|\hat{r}_{k}\right\| /\left\|r_{0}\right\|<\delta \text { or }\left\|\hat{r}_{k}\right\|<\text { tol then } \\
& \text { for } l \leftarrow k-1 \text { down to } 0 \text { do } \\
& \text { solve } \gamma_{l} \chi_{l}+\sum_{i=l+1}^{k-1} \beta_{l, i} \chi_{i}=\alpha_{l} \text { for } \chi_{l} \\
& \text { end } \\
& \hat{x}=\sum_{i=0}^{k-1} \chi_{i} \hat{p}_{i} \\
& x=x+\hat{x} \\
& r_{0}=b^{\prime}-A x \\
& \hat{r}_{0}=r_{0} \\
& \hat{x}=0 \\
& k=0 \\
& \text { end } \\
& \text { end }
\end{aligned}
$$

Algorithm 1: Mixed-precision GCR-DD solver. Lowprecision fields are indicated with a hat $\left({ }^{\wedge}\right)$; e.g., $x$ and $\hat{x}$ correspond to high- and low-precision respectively. The domain-decomposed preconditioner matrix is denoted by $K$, and the desired solver tolerance is given by tol. The parameter $k_{\max }$ denotes the maximum size of the Krylov subspace.

ing that using a mixed-precision strategy is not possible; the extra BLAS1-type linear algebra incurred is extremely bandwidth intensive and so can reduce performance significantly; multi-shift solvers come with much larger memory requirements since one has to keep both the $N$ solution and direction vectors in memory.

With these restrictions in mind, we have employed a modified multi-shift solver strategy where we solve Equation (4) using a pure single-precision multi-shift CG solver and then use mixed-precision sequential CG, refining each of the $x_{i}$ solution vectors until the desired tolerance has been reached ${ }^{3}$ This allows us to perform most of the operations in singleprecision arithmetic while still achieving double-precision accuracy. Since double precision is not introduced until after the multi-shift solver is completed, the memory requirements are much lower than if a pure double-precision multi-shift solver were employed, allowing the problem to be solved on

\footnotetext{
${ }^{3}$ Unfortunately, such an algorithm is not amenable to the use of half precision since the solutions produced from the initial multi-shift solver would be too inaccurate, demanding a large degree of correction in the subsequent sequential refinements.
} 
a smaller number of GPUs. When compared to doing just sequential mixed-precision $\mathrm{CG}$, the sustained performance measured in flops is significantly lower because of the increased linear algebra; however, the time to solution is significantly shorter.

\section{SOLVER PERFORMANCE RESULTS}

\subsection{Wilson-clover}

Our Wilson-Clover solver benchmarks were run with the QUDA library being driven by the Chroma 25 code. The solves were performed on a lattice of volume $32^{3} \times 256$ lattice sites from a recent large scale production run, spanning several facilities including Cray machines at NICS and OLCF, as well as BlueGene/L facilities at LLNL and a BlueGene/P facility at Argonne National Laboratory (ANL). The quark mass used in the generation of the configuration corresponds to a pion mass of $\simeq 230 \mathrm{MeV}$ in physical units 26 .

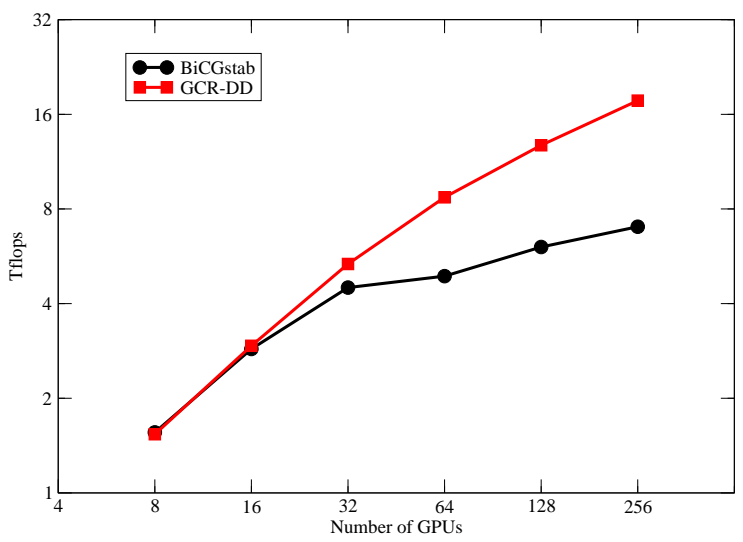

Figure 7: Sustained strong-scaling performance in Gflops of the Wilson-clover mixed precision BiCGstab and GCR-DD solvers $\left(V=32^{3} \times 256,10\right.$ steps of MR used to evaluate the preconditioner).

In Fig. 7, we plot the sustained performance in Tflops of both the BiCGstab and GCR-DD solvers. For the BiCGstab solver, we can see that despite the multi-dimensional parallelization, we are unable to effectively scale past 32 GPUs because of the increased surface-to-volume ratio. The GCRDD solver does not suffer from such problems and scales to 256 GPUs. As described above, the raw flop count is not a good metric of actual speed since the the iteration count is a function of the local block size. In Fig. 8 we compare the actual time to solution between the two solvers. While at 32 GPUs BiCGstab is a superior solver, past this point GCR-DD exhibits significantly reduced time to solution, improving performance over BiCGstab by $1.52 \mathrm{x}, 1.63 \mathrm{x}$, and $1.64 \mathrm{x}$ at 64,128 , and 256 GPUs respectively. Despite the improvement in scaling, we see that at 256 GPUs we have reached the limit of this algorithm. While we have vastly reduced communication overhead by switching to GCR-DD, there is still a significant fraction of the computation that requires full communication. This causes an Amdahl's law effect to come into play, which is demonstrated by the fact that the slope of the slow down for GCR and BiCGstab is identical in moving from 128 to 256 GPUs. Additionally, we note that if we perform a single-GPU run with the same per-GPU volume as considered here for 256 GPUs, performance is almost a factor of two slower than that for a run corresponding to 16 GPUs. Presumably this is due to the GPU not being completely saturated at this small problem size.

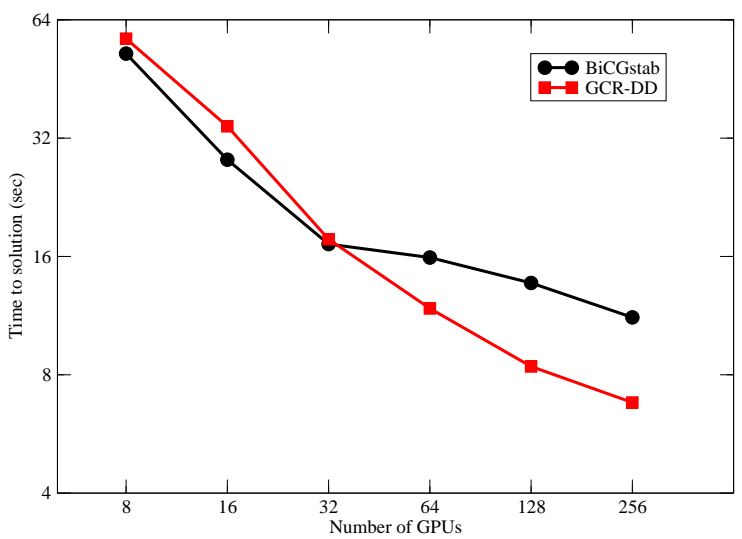

Figure 8: Sustained strong-scaling time to solution in seconds of the Wilson-clover mixed precision BiCGstab and GCR-DD solvers $\left(V=32^{3} \times 256,10\right.$ steps of MR used to evaluate the preconditioner).

In terms of raw performance, it can be seen that the GCRDD solver achieves greater than 10 Tflops on partitions of 128 GPUs and above. Thinking more conservatively, one can use the improvement factors in the time to solution between BiCGStab and GCR to assign an "effective BiCGStab performance" number to the GCR solves. On 128 GPUs GCR performs as if it were BiCGStab running at 9.95 Tflops, whereas on 256 GPUs it is as if it were BiCGStab running at 11.5 Tflops. To put the performance results reported here into perspective, in Fig. 9 we show a strong-scaling benchmark from a variety of leadership computing systems on a lattice of the same volume as used here. Results are shown for the Jaguar Cray XT4 (recently retired) and Jaguar PF Cray XT5 systems at OLCF, as well as the Intrepid BlueGene/P facility at ANL. The performance range of 10-17 Tflops is attained on partitions of size greater than 16,384 cores on all these systems. Hence, we believe it is fair to say that the results obtained in this work are on par with capability-class systems.

\subsection{Improved staggered}

The results for improved staggered fermions were obtained using the QUDA library driven by the publicly available MIMD Lattice Collaboration (MILC) code 27. The $64^{3} \times$ 192 gauge fields used for this study corresponds to a pion mass of $\simeq 320 \mathrm{MeV}$ in physical units 28. 


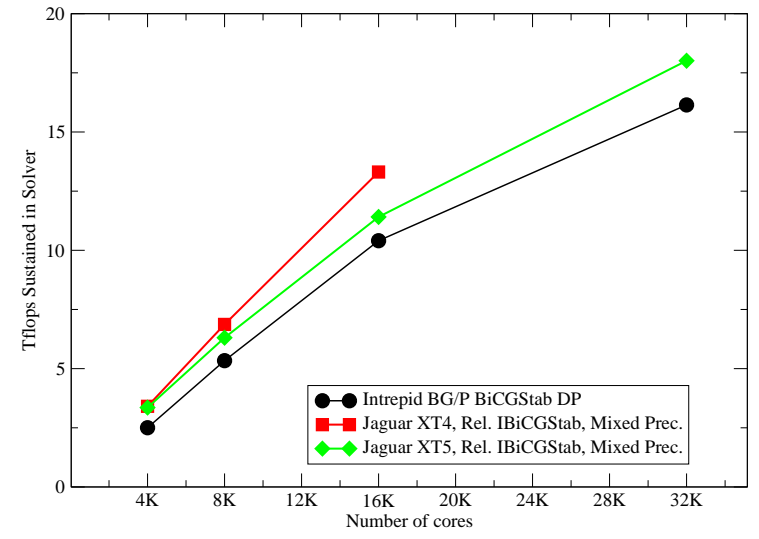

Figure 9: Strong-scaling benchmarks on a lattice of size $32^{3} \times 256$ from Cray XT4 (Jaguar), Cray XT5 (JaguarPF) and BlueGene/P (Intrepid). Solves were done to double-precision accuracy. The Cray solvers used mixed (double-single) precision; the BG/P solver used pure double precision.

In Fig. 10, we plot the performance of the mixed-precision multi-shift CG algorithm. When running the full solver, the minimum number of GPUs that can accommodate the task is 64 . Reasonable strong scaling is observed, where we achieve a speed-up of $2.56 \mathrm{x}$ in moving from 64 to 256 GPUs.

With 256 GPUs, we achieve 5.49 Tflops with double-single mixed precision. In Ref. [6], we observed an approximately $20 \%$ increase in iteration count for the mixed precision solver, comparing to the pure double precision one. To put this in perspective, the CPU version of MILC running on Kraken, a Cray XT5 system housed at NICS, achieves 942 Gflops with 4096 CPU cores for the double precision multi-shift solver. This means one GPU computes approximately as fast as 74 CPU cores in large-scale runs.

\section{CONCLUSIONS}

Our main result is to demonstrate that by the use of multidimensionsional parallelization and an additive Schwarz preconditioner, the Wilson-clover solver for lattice QCD can be successfully strong-scaled to over 100 GPUs. This is a significant achievement demonstrating that GPU clusters are capable of delivering in excess of 10 teraflops of performance, a minimal capability required to apply GPU clusters to the generation of lattice ensembles. Additionally, multidimensional parallelization has enabled the use of GPUs for asqtad solvers at leading-edge lattice volumes, a feat which was previously not possible because of the decreased locality of the asqtad operator.

Clearly, the present use of a simple non-overlapping additive Schwarz preconditioner is only a first step. It is very likely that more sophisticated methods with overlapping domains or multiple levels of Schwarz-type blocking to take advantage of the multiple levels of memory locality that a GPU cluster offers can be devised to improve the scaling

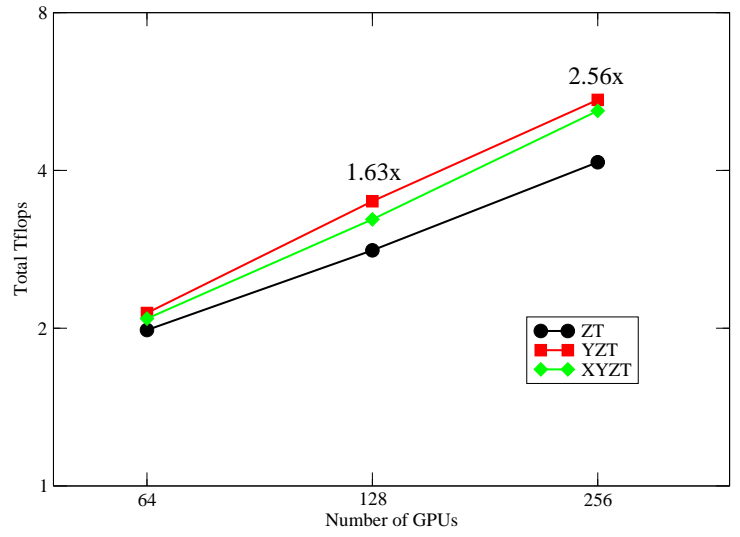

Figure 10: Sustained strong-scaling performance in Gflops of the asqtad mixed-precision multi-shift solver. The legend labels denote which dimensions are partitioned between GPUs $\left(V=64^{3} \times 192\right)$.

substantially. Moreover, we view GPUs and the use of the Schwarz preconditioner as parts of a larger restructuring of algorithms and software to address the inevitable future of heterogeneous architectures with deep memory hierarchies. We anticipate that the arsenal of tools needed for the future of lattice QCD and similarly structured problems (e.g., finite difference problems, material simulations, etc.) at the exascale will include domain decomposition, mixed-precision solvers and data compression/recomputation strategies.

\section{ACKNOWLEDGMENTS}

We gratefully acknowledge the use of the Edge GPU cluster at Lawrence Livermore National Laboratory. This work was supported in part by NSF grants OCI-0946441, OCI1060012, OCI-1060067, and PHY-0555234, as well as DOE grants DE-FC02-06ER41439, DE-FC02-06ER41440, DE-FC0206ER41443, DE-FG02-91ER40661, and DE-FG02-91ER40676. BJ additionally acknowledges support under DOE grant DEAC05-06OR23177, under which Jefferson Science Associates LLC manages and operates Jefferson Lab. GS is funded through the Institute for Advanced Computing Applications and Technologies (IACAT) at the University of Illinois at Urbana-Champaign. The U.S. Government retains a nonexclusive, paid-up, irrevocable, world-wide license to publish or reproduce this manuscript for U.S. Government purposes.

\section{REFERENCES}

[1] http://en.wikipedia.org/wiki/Grand_Challenge, 2011.

[2] http://www.research.ibm.com/bluegene/BG_ External_Presentation_January_2002.pdf, 2002.

[3] M. A. Clark, R. Babich, K. Barros, R. C. Brower, and C. Rebbi, "Solving Lattice QCD systems of equations using mixed precision solvers on GPUs," Comput. Phys. Commun. 181 (2010) 1517-1528 arXiv:0911.3191 [hep-lat]. 
[4] R. Babich, M. A. Clark, and B. Joó, "Parallelizing the QUDA Library for Multi-GPU Calculations in Lattice Quantum Chromodynamics," in Proceedings of the 2010 ACM/IEEE International Conference for High Performance Computing, Networking, Storage and Analysis, SC '10, pp. 1-11. IEEE Computer Society, Washington, DC, USA, 2010. arXiv:1011.0024 [hep-lat].

[5] S. Gottlieb, G. Shi, A. Torok, and V. Kindratenko, "QUDA programming for staggered quarks," $P o S$ LATTICE2010 (2010) 026.

[6] G. Shi, S. Gottlieb, A. Torok, and V. V. Kindratenko, "Design of MILC lattice QCD application for GPU clusters," in IPDPS. IEEE, 2011.

[7] B. Sheikholeslami and R. Wohlert, "Improved Continuum Limit Lattice Action for QCD with Wilson Fermions," Nucl. Phys. B259 (1985) 572 .

[8] A. Bazavov, D. Toussaint, C. Bernard, J. Laiho, C. DeTar, L. Levkova, M. B. Oktay, S. Gottlieb, U. M. Heller, J. E. Hetrick, P. B. Mackenzie, R. Sugar, and R. S. Van de Water, "Nonperturbative QCD simulations with $2+1$ flavors of improved staggered quarks," Rev. Mod. Phys. 82 no. 2, (May, 2010) 1349-1417.

[9] M. R. Hestenes and E. Stiefel, "Methods of Conjugate Gradients for Solving Linear Systems," Journal of Research of the National Bureau of Standards 49 no. 6, (Dec., 1952) 409-436.

[10] H. A. van der Vorst, "Bi-CGSTAB: A Fast and Smoothly Converging Variant of Bi-CG for the Solution of Nonsymmetric Linear Systems," SIAM Journal on Scientific and Statistical Computing 13 no. 2, (1992) 631-644.

[11] T. A. Degrand and P. Rossi, "Conditioning techniques for dynamical fermions," Computer Physics Communications 60 no. 2, (1990) $211-214$

[12] B. Jegerlehner, "Krylov space solvers for shifted linear systems," arXiv:hep-lat/9612014

[13] H. A. Schwarz, "Über einen Grenzübergang durch alternierendes Verfahren," Vierteljahrsschrift der Naturforschenden Gesellschaft in Zürich 15 (1870) 272-286.

[14] G. I. Egri, Z. Fodor, C. Hoelbling, S. D. Katz, D. Nógrádi, and K. K. Szabó, "Lattice QCD as a video game," Computer Physics Communications 177 no. 8, (2007) 631 - 639, arXiv:0611022 [hep-lat].

[15] M. A. Clark, "QCD on GPUs: cost effective supercomputing," PoS LATTICE2009 (2009) 003.

[16] A. Alexandru, C. Pelissier, B. Gamari, and F. Lee, "Multi-mass solvers for lattice QCD on GPUs," arXiv:1103.5103 [hep-lat].

[17] TWQCD Collaboration, T.-W. Chiu, T.-H. Hsieh, Y.-Y. Mao, and K. Ogawa, "GPU-Based Conjugate Gradient Solver for Lattice QCD with Domain-Wall Fermions," PoS LATTICE2010 (2010) 030, arXiv:1101.0423 [hep-lat].

[18] A. Alexandru, M. Lujan, C. Pelissier, B. Gamari, and F. X. Lee, "Efficient implementation of the overlap operator on multi- GPUs," arXiv:1106.4964 [hep-lat].

[19] S. Borsáni, "Thermodynamics from accelerated architectures." http://crunch.ikp.physik.tu-darmstadt.de/ gpu2011/Talks/Borsanyi_Darmstadt_GPU.pdf, 2011.

[20] M. Luscher, "Solution of the Dirac equation in lattice QCD using a domain decomposition method," Comput.Phys.Commun. 156 (2004) 209-220, arXiv:hep-lat/0310048 [hep-lat].

[21] Y. Osaki and K.-I. Ishikawa, "Domain Decomposition method on GPU cluster," PoS LATTICE2010 (2010) 036, arXiv:1011.3318 [hep-lat].

[22] http://lattice.github.com/quda 2011.

[23] G. Ruetsch and P. Micikevicius, "Optimizing matrix transpose in CUDA," NVIDIA Technical Report (2009) .

[24] http://www.mellanox.com/pdf/whitepapers/TB_ GPU_Direct.pdf 2010.

[25] R. G. Edwards and B. Joó, "The Chroma software system for lattice QCD," Nucl. Phys. Proc. Suppl. 140 (2005) 832, arXiv:hep-lat/0409003.

[26] H.-W. Lin et al., "First results from $2+1$ dynamical quark flavors on an anisotropic lattice: light-hadron spectroscopy and setting the strange-quark mass," Phys. Rev. D79 (2009) 034502, arXiv:0810.3588 [hep-ph]

[27] MIMD Lattice Collaboration, C. Bernard et al., "The MILC Code." http: //www.physics.utah.edu/ detar/milc/milcv7.pdf 2010.

[28] A. Bazavov, D. Toussaint, C. Bernard, J. Laiho, C. DeTar, et al., "Nonperturbative QCD simulations with $2+1$ flavors of improved staggered quarks," Rev.Mod.Phys. 82 (2010) 1349-1417, arXiv:0903.3598 [hep-lat]. 